\title{
Mitteilungen
}

aus der pharmazeutischen $\Delta$ bteilung des chemischen Instituts der westfalischen Wilhelms. Universitat zu Munster i. W.

\section{Ueber die Verwertung der Samenkerno von Prunus domestica L.}

Von. Georg Ka B ner.

(Eingegangen den 31. I. 1918.)

Obwohl nach Ausweis des Schrifttums bereits von anderen Autoren Angaben über die Verwendung der Obstkerne, zumal derer von Prunus domestica, nicht allein zur Herstellung von fettem Oel, sondern auch zur Gewinnung eines blausäurehaltigen Destillats, welches dem Aqua Amygdalarum amararum bzw. dem Aqua Lauracerasi völlig gleichwertig ist, gemacht wurden, hielt ich es doch nicht für überflüssig, besonders im Hinblick auf die gegenwärtigen Zeitumstände, abermals eine solche Verarbeitung durchzufuhren, uber deren Ergebnisse ich hiermit berichte.

Es wurde eine größere Anzahl Kerne aus reifen, 1916 geernteten Früchten der Hauspflaume auf mechanische Weise oder durch Einweichen in Wasser und mehrtïgiges Vergären darin aller Fleischreste entkleidet und nach sorgfältigem $\mathrm{Abwaschen} \mathrm{in} \mathrm{sauberem} \mathrm{Zustande} \mathrm{ge-}$ wonnen und getrocknet. Dieselben wurden durch Zerschlagen der Steinschalen geöffnet und die in ihnen enthaltenen Samenkerne durch Auslesen mit der Hand gesammelt. Man hätte ja auch das von Alper ${ }^{1}$ ) vorgeschlagene Trennungs- und Sortiêrverfahren, bei welchem eine auf das spezifische Gewicht von 1,15 gebrachte Chlorcalcium- oder Magnesiumchlorid-Lauge zur mechanischen Scheidung ron Schalen und Kernen dient, benutzen können, doch lag mir daran, eine Einwirkung fremder Stoffe auf die Substanzen der Kerne möglichst auszuschließen. Im Großen wird man wohl freilich schon aus wirtschaftlichen Gründen zu einem derartigen mechanischen Verfahren (eventuell auch einem Windseparier- oder ähnlichen Verfahren) greifen müssen, da das Zertrummern der Steine und das Auslesen der Oelkerne mit der Hand recht zeitraubend ist.

1) Chemiker-Zeitung, 40. Jahrg. 8. 846, 1916. 
Es wurden $850 \mathrm{~g}$ sauberer trockener Samenkerno von hellbrauner Farbe gewonnen, deren_Testa durch Eintrocknen runzlich erschien.

\section{Die Gewinnung des Petten Oeles.}

Die Samenkerne mußten zum Zwecke der Oelgewinnung zerkleinert werden. Das Mahlen der fettigen Kerne in einer Laboratoriumsmühle gab wegen Verschmierens der Mahlflächen kein bofriedigendes Resultat, so daß nichts weiter ubrig blieb, als sie im Mörser zu zerstampfen und das erhaltene Pulver durch ein mittelfeines Sieb No. 4 abzusieben. Eine vorherige Entschälung, wie sie wohl bei der Verarbeitung der viel fleischigeren Mandeln ublich ist, fand nicht statt. Das Pulver aah daher auch nicht schneeweiB, sondern weißlichbraun aus; die Ausbeute betrug $830 \mathrm{~g}$, so daB beim Zrrkleinern und Sieben nur ein Verlust von $20 \mathrm{~g}$ entstanden war. ... $825 \mathrm{~g}$ Semenpulver wurde nun in fünf einzelnen Portionen in ein tariertes Preßtuch eingeschlagen und jede Portion fur sich in einer hydraulischen Presse einem Druck bis zu 350 Atmosphären auggesetzt. Die Ausbeute an direkt gewonnenem Oel betrug 179,5 g.

In dem Preßtuch waren verblieben . . . . . . 22.5 g,

so daß also aus den $825 \mathrm{~g}$ Samenkernpulver . . . . . 202,0 g] Oel erhalten wurden oder $24,48 \%$ vom Gewicht der Samen.

Da nun laut einer früheren Analyse') auf dem Wege der Extraktion der Samenkerne mittels Aether aus allerdings besonders schönen Pflaumen des Jahres 1913 ein Betrag von $42,92 \%$ Oel erhalten worden war, so würden somit in den Preßkuchen etwa noch $17,44 \%$ Oel zurückgehalten sein oder $40,06 \%$ des Gesamtgehalts, so daß nur rund $\%$ des Oeles durch Pressen gewonnen wurden.

Neuerdings hat auch $\mathrm{D}_{\mathrm{a}} \mathrm{r} \mathbf{\mathrm { a }} \mathrm{s}^{2}$ ) die aus Pflaumenkernen $\mathrm{zu}$ erhaltenden Produkte beschrieben und seine Ergebnis̀se veröffentlicht. Er erbielt aus $5 \mathrm{~kg}$ Pflaumenkernen durch Pressen durchschnittlich $200 \mathrm{~g}$ fettes Oel. Da nach meiner Bestimmung aus 2,544 $\mathrm{g}$ Steinkernen $0,46 \mathrm{~g}$ Samenkerne und aus den Samen seinerzeit durch Extraktion 42,92\% Oel erhalten wurden, berechnet sich die Ausbeute von $D$ a $r$ v s auf rund $50 \%$ des in den Samen enthaltenen Oeles, während ich, wie eben gezeigt, mit Hilfé der hydraulischen Presse rund 60\% oder nach Abzug des im Preßtuch ver-

3) Dieses Archiv Bd. 252, s. 403, 1814.

1) Ztachr. d. Allg. österr. Apoth.-Ver. 1916, No. 62. F r u nz Darva s, Die Erschließung und Verwertung der Pflaumenkerno. 
bliebenen, dooh nicht verlorenen Anteil bentells rund $50 \%$ des Oeler gewonnen hatte:

\section{Beschaffenheit und Eigenscharten des Oeles.}

Das durch Pressen aus gesunden reifen Samenkernen von Prunus domestic. erhaltene Oel war von goldgelber Farbe und zeigte in $\mathrm{A} \mathrm{b} \mathrm{b} \mathrm{e's} \mathrm{Refraktometer} \mathrm{untersucht} \mathrm{den} \mathrm{Wert} \mathrm{n}_{\mathrm{D}}=\mathbf{1 , 4 7 0 5}$ bei $20^{\circ} \mathrm{C}$., während in dem früher (1. c.) durch Extraktion gewonnenen Oel der Wert $n_{D}=1,4715$ gefunden wurde.

Die Jodzahl betrug 104,9.

Bei der großen Aehnlichkeit des Ol. Pruni mit Ol. Amygdal. schien es mir wichtig, das Verhalten des Oeles beim Abkuhlen auf niedere Temperaturen kennen zu lernen. Es wurde daher eine kleine Probe desselben in einem trockenen Reagenzglas in eine Kältemischung von Eis und Kochsala gebracht und anhaltend mit einem Thermometer gerührt.

Erst bei $-15^{\circ} \mathrm{C}$. schieden sich aus dem Oel einige krystallinische Flocken ab, es wurde im übrigen nur etwas dickflüssiger. Das früher durch Extraktion erhaltene Oel blieb dagegen auch bei dieser niedrigen Temperatur noch klar.

Diese Probe zeigt deutlicher und schneller wie die sonst zur Kritik herangezogenen Konstanten die außerordentliche Verwandtschaft und Aehnlichkeit des Pflaumkernöles mit dem fetten Mandelöl an, von welchem das Deutsche Arzneibuch ein Flussigbleiben bei nur $-10^{\circ} \mathrm{C}$. verlangt.

\section{Die Herstellung von Aqua Pruni.}

Die Preßrückstände, im Gewicht von $623 \mathrm{~g}$, wurden zerkleinert und nach der für Herstellung des Bittermandelwassers angegebenen Vorschrift des D. A:-B. 5 behandelt, wobei allerdings dem Minderbetrag der Samenkerne an Amygdalin gegenüber dem Gehalt der bitteren Mandeln Rechnung getragen wurde. Da gemäß früherer Bestimmung ${ }^{1}$ ) der Amygdalingehalt der Samen von Prunus domestica. $1,82 \%$ beträgt, derjenige von Amygdal. amar. in maximo 3,5\%, so wurden nur 41/2 Teile Hauptdestillat, statt der 9 . Teile der Vorschrift für Aqua amygdal. amar. zu erhalten gesucht. Das Ergebnis zeigte, daß diese Abweichung berechtigt war.

Der dem Destillat zu machende Alkoholzusatz betrug demgemäß auch nur die Hälfte, also statt 3 nur $1 \frac{1}{2}$ Teile, prozentualiter

5) Dieses Archiv Bul 252. 3 . 40.5. 
indessen abensoriel wie in Aq. auygdal. Im einzelnen war die Behandlung folgende: Das aurgepreBte Samenkernpulver wurde in einen geräumigen Glaskolben gebracht und znnüchst mit den rorgeschriebenem 20 Teilen Wasser, d. h. 1375 g (wie der Ansatz $12: 20$ $=825: \mathrm{x}$ ergibt) auf 12 Teile Pulver cingemaisht und fast 24 Stunden (statt 12 der Vorschrift) stehen gelassen. Jie Masse besal nach dieser Zeit sinen äuBerst kräftigen Bittermandelol- und Blausänregeruch.

Da sie aber durchaus nicht breiförmig, sondern mehr stückig; und krümelig war, so wurden noch $1000 \mathrm{~g}$ Wasser zugesetzt, da sonst große Schwierigkeiten boi der Destillation zu befürchten waren.

Im Groben fallen diese ja fort, wenn man in gat verzinnten Metallopparaten und mit gespanntem Wascerdampf irbejtet. Hier aber mußte an die Möglichkeit des Anbrennens der breiförmigen Substanz an dem Boden des Glasgefäßes und des Zerspringens desselben gedacht werden. Un dieses zu verhïten, wurde der Kolben in ein Paraffinbad und dieses witder in cin Sandbad gestellt.

Der Kolben war mit einem doppelt durchbohrten Kork tojfen versehen, durch dessen cine Bohrung ein Glasrohr zum Ei.leiten von Wasserdampf bis nahe zum Bóden des Kolbens reichte, durch dessen andere ein in spitzem Winkel gebogenes Glasrohr den Dampf zur Verdichtung nach cinem Liebig'schen Kühler und der damit verbundenen Vorlage führte, nachdem er das Destillationsgut durchdrungen hatte.

Die Destillation ging bei dieser Vorsicht unter Einleiten ron Dampf glatt von statten. Es wurden in gewogenen Vorlagen 3 Destillate aufgefangen, und zwar in

Vorlage 1 in welche $103,1 \&$ Alkohol gebracht waren

$$
375 \mathrm{~g} \text { (denn } 12: 1,5=825: \times x=103,1)
$$

Vorlage II $100 \mathrm{~g}$ und

Vorlage III $65 \mathrm{~g}$.

Bei der durch Titration mit Silberlösung ausgeführten Intersuchung ergub

Destillat J einen Gehalt von 0,46575 \& Blausäure.

Destillat II einen Gehalt von $0, C 4860 \mathrm{~g}$ Blausäure.

Destillat IIT einen Gchalt von $0.01755 \mathrm{~g}$ Bldusäure. also zusammen $0,53190 \mathrm{~g}$ Blausäure.

Die nach Beiscitostellung des Destillat; III noch kurze Zt it fortgesetzte Dostillation ließ bei der Prufung des Kondensats mit Silberlösung nach rorherigem Zusatz ron Ammoniak und dgmm 
Uebersättigen mit Salpetersäure nach immer einen, wentr auch schwachen Blausäuregehalt erkennen, doch lohnte offenbar das Sammeln weiterer Nachläufe nicht, es sei denn, daß man hätte auf diesem Wege quantitativ den Blausäuregehalt und damit auch den an Amygdalin hätte bestimmen wollen, was ich bei Anwendung der nötigen Vorsichtsmaßregeln nicht für aussichtslos halte, da unbeschadet kleiner Verluste durch Zurückhalten von Benzaldehyd im Restbetrag an fettem Ocl, durch damit ermöglichte Hydrolyse eines kleinen Teils der Blansäure, durch Festlegung solcher auch durch den Kalkgehalt des Einmaischwasser's (we.halb besser destilliertes Wasser zum Anrühren der Preßkuchen zu nehmen ist) und durch sonstige Nebenreabtionen ein annähernd dem seinerzeit gefundenen Amygdalin-Gehalt entsprechender Blausäuregehalt zu finden sein dürfte. Berechnet man nämlich die aus $1,8 \%$ Amygdalin zu erwartende Blausauremenge, so miiliten aus $825 \mathrm{~g}$ Samenkerne von Prunus domestica $0,78 \mathrm{~g} \mathrm{HCN}$ zu erhalten sein, während ich in den 3 verwertbaren Destillaten rund $0,532 \mathrm{~g}$ wiederfand. Jedenfalls ergibt sich auch schon durch die ohne Absicht des quantitativen Wiedergewinns des Blausiure durchgeführte Destillation, daß der Amygdalingehalt der Samenkerne grölBer ist als man bisher wohl annahm und im Durchschnitt nicht wesentlich unter $1,8 \%$ liegen wird.

Da r va s (l. c.) gewann aus j̃ $\mathrm{kg}$ Steinkernen $670 \mathrm{~g}$ Destillat mit 1,35\% Blausäure $=0,9045 \mathrm{~g}$ HCN. Tach dieser Ausbeute berechnet sich die aus den von mir verwendeten $825 \mathrm{~g}$ Samenkernpulver zu erhaltende Blausüure auf :ogar $0,81 \mathrm{~g}$, liegt also noch etwas über dem durch Rechnung gefundenen Wert 0,78. Auch das von $\mathbf{D}$ a $\mathrm{r}$ v a s erhaltene Resultat bestätigt die durch die frühere Arbeit (l. c. S. 405) gewonnene Ansicht über den Amygdalin-Gehalt der Samen.

Damit man die ihm entsprechende Menge Blausäure voll erhalten kann, wird es notwendig 'sein, die mit Wasser hergestellte Maische nicht länger als erforderlich ist, stehen zu lassen, dann flott im Dampfstrome zu destillieren ụnd im übrigen während der Destillation für ein Umrühren und gutes Durchmischen des Samenkernbreies Sorge zu tragen.

Nachdem die Destillation durchgeführt war, bemerkte ich bei dem Auseinandernehmen des Apparats, daß im Liebig'schen Kühler noch einige Tropfen krystallinisch erstarrter öliger Substanz zurückgeblieben waren, welche bei der Untersuchung nur Spuren von Blausäure enthielten und offenbar aus Benzoln, dem Poly. merisationsprodukt von Benzaldehyd, bestanden, $2 \mathrm{C}_{6} \mathrm{H}_{5}-\mathrm{CHO}$ 
$=\mathrm{C}_{6} \mathrm{H}_{5} \mathrm{CH} \cdot \mathrm{OH}-\mathrm{CO} \cdot \mathrm{C}_{6} \mathrm{H}_{5}$, dessen Entstehung an dem beob. achteten Orte mit ein Beweis dafur ist, da $B$ die Vereinigung von Benzaldehyd und Blausäure zu Benzaldehydcyanhydrin wesentlich erst im Destillat stattfindet.

Nach der Gehaltsbestimmung der Destillate durch Titration mit $1 / 10-\mathrm{N}$. Silbernitrat-Lösung waren

von I noch vorhanden $345 \mathrm{~g}$ mit $0,42489 \mathrm{~g}$ HCN
von II noch vorhanden $90 \mathrm{~g}$ mit $0,04374 \mathrm{~g} \mathrm{HCN}$
von III noch vorhanden $55 \mathrm{~g}$ mit $0,014,85 \mathrm{~g}$ HCN.

Die Summe von I und II ergibt $43 a \mathrm{~g}$ mit einem Gehalt von $0,47223 \mathrm{~g}$ HCN, beide Destillate wurden gemischt and durch Zusatz

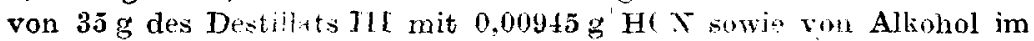

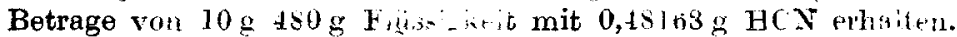

Ditses Arma pruni enthielt demnach einen Blausäuregehalt von 1,003 pro Miite.

Es besa $B$ ganz den Geruch und Geschmack des Bittermandelwassers und entsprach den Anforderungen des D. A.-B. 5 für Aqua amygdal. am., insbesondere lag auch der Gehalt an freier, nicht an Benzaldehyd gebundener Blatsäure unterhalb der vom D. A.-B. 5 als zulässig festgelegten Grenze.

\section{Die Verwertung des Rückstandes.}

Der nach der Herstellung des Aqua Pruni verbliebene Destillationsrückstand besa $B$ noch rund $40 \%$ des Oelgehalts der Sảmen, enthielt also für die angewandte Menge von $825 \mathrm{~g}$ noch rund $141 \mathrm{~g}$ fettes Oel; außerdem schloß er das gesamte Eiweiß der Samen ein (nach früherer Untersuchung 47,18\% der fettfreien Substanz) und andere Stoffe (Zucker, Salze). Da bei der bisherigen Ver- arbeitung keine das Aussehen, die Güte und den Nährwert des Materials beeinträch tigenden Manipulationen vorgenommen wurden; so lag es nahe, den Rückstand noch für Zwecke der menschlichen Ernährung auszunützen.

Besonderen Anreiz dazu bot der Umstand, daß dem Brei noch ein kleiner Betrag an Benzialdehyd verblieben war, welcher ihm einen angenehmen aromatischen Geschmack verlieh.

Er wurde deshalb aus dem Destillationskolben herausgebracht und der Küche des Haushalts überwiesen, welche mit Zusatz von otwas Mehl, Zucker, Salz und Lockerungsmittel daraus ein Gebäck herstellte, das durch Wohlgeschmack und Bekömmlichkeit allgemeinen Anklang fand. 
Unter Zusammenfassurg des Inhalts vorstehender Mitteilungen, muß es als empfehlenswert angesehen werden, mit Hilfe einfacher Vorrichtungen des Laboratoriums (Presse, Destillierblase) eine restlose Aufarbeitung der Pflaumenkerne, wo sie in hinreichender Menge vorhanden sind und leicht von der Steinschale befreit werden können, vorzunehmen, da hierbei an pharmazeutisch wichtigen Produkten erhalten werden: rund 20\% (vom Gewicht der Samen) fettes Oel, welches dem fetten Oel der Mandeln sehr ähnlich ist, ferner $50-80 \%$ blausäurehaltiges Destillat (Aq. Pruni als evtl: Ersatz für Aq. Amygdal. amar.) sowie ein öl- und eiweißreicher Rückstand, welcher für Speisezwecke gut verwertbar ist.

\section{Gasmessungen mit einer gewöhnlichen Burette zur Bestimmung von Harnstoff im Harn und von Aethylnitrit im Spiritus Aetheris nitrosi.}

Von G. Frerichs und E. Mannhe im - Bonn.

(Eingegangen den 1. IT. 1918.)

\section{Bestimmung von Harnstoff.}

Zur Bestimmung des Harnstoffs benutzt man bekanntlich die Abscheidung des Stickstoffs aus dem Harnstoff durch Einwirkung von $\mathbf{N a t r i u m h y o b r o m i t :}$

$$
\mathrm{CO}\left(\mathrm{NH}_{2}\right)_{2}+3 \mathrm{NaOBr}=\mathrm{CO}_{2}+2 \mathrm{H}_{2} \mathrm{O}+3 \mathrm{NaBr}+\mathrm{N}_{2} \text {. }
$$

Zur Messung des Stickstoffs werden verschiedene Apparate benutzt, z. B. das Nitrometer von $\mathrm{Lunge}$ und einige andere, besonders für die Bestimmung des Harnstoffs im Harn bestimmte Apparate, die alle das gemeinsam haben, da B sie ziemlich teuer sind. Man kann nun sehr leicht aus den in jedem Laboratorium vorhandenen Geräten einen Apparat zusammenstellen, der genau die gleichen Dienste leistet wie die übrigen Apparate. Als Me B $\mathrm{r}$ oh $\mathrm{r}$ für den Stickstoff wird eine $\mathrm{ge}$-w öh $\mathrm{nlich}$ e $\mathrm{B}$ ü $\mathrm{r}$ e t te $\mathrm{A}$ von $50 \mathrm{ccm}$ Inhalt ohne Hahn benutzt, die, wie die Zeichnung Fig. 1 wiedergibt, durch einen Schlauch (etwa $60-70 \mathrm{~cm}$ lang) mit eine 


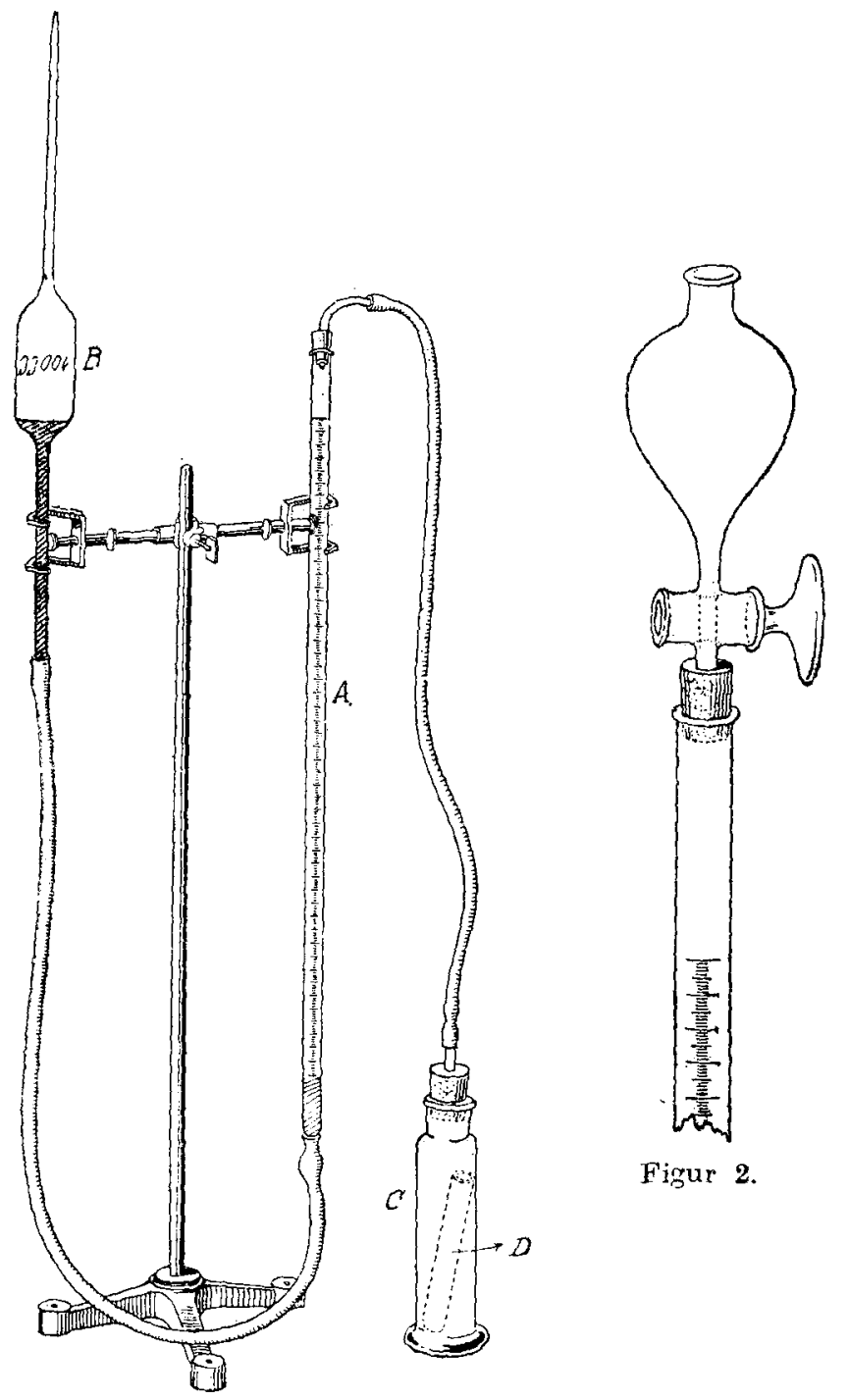

Figur 1. 